\title{
Prospective Cohort Study Assessing the Use of Peripheral Saphenous Venous Pressure Monitoring as a Marker of the Transcaval Venous Pressure Gradient in Liver Transplant Surgery
}

\author{
Jeremy Fabes, ${ }^{1,2}$ Michael Spiro, 1,3 on behalf of the Royal Free Perioperative Research Group ${ }^{1,3}$
}

\begin{abstract}
Objectives: Assessment of the transcaval venous pressure gradient, the central venous to inferior vena caval pressure, assists anesthetists and surgeons in management of liver transplant recipients. Traditionally, this entails insertion of a femoral central line with increased patient risk and health care cost. Here, we assessed the ability of a saphenous vein cannula to act as a surrogate for the femoral central line as a means to assess the transcaval pressure gradient in a safer and less invasive manner.

Materials and Methods: A prospective cohort of 22 patients undergoing liver transplant underwent saphenous vein cannulation in addition to insertion of a femoral and internal jugular central venous catheter. Data were collected throughout each phase of surgery to assess the central, femoral, and saphenous vein pressures; results of a range of relevant physiological and ventilatory data were also collected.

Results: The primary outcome, the correlation between saphenous and femoral venous pressure throughout surgery, was acceptable $\left(r^{2}=0.491, P<.001\right)$. During the anhepatic phase of surgery, this correlation improved $\left(r^{2}=0.912, P<.001\right)$. The correlation between the femoral to central venous pressure and saphenous to central venous pressure gradients was also reasonable throughout surgery $\left(r^{2}=0.386, P<.001\right)$, and this correlation was significantly stronger during the anhepatic phase $\left(r^{2}=0.935, P<.001\right)$.
\end{abstract}

From the ${ }^{1}$ Department of Anaesthesia, The Royal Free NHS Foundation Trust, London; the ${ }^{2}$ Faculty of Health, University of Plymouth, Plymouth; and the ${ }^{3}$ Royal Free Perioperative Research Group, London, United Kingdom

Acknowledgements: The Royal Free Perioperative Research Group includes D. Jackson, N. Schofield, C. Melikian, D. Krzanicki, S. Rahman, S. Harrison, J. Prout, J. Cooper, C. Morkane,

D. Milliken, J. Benham-Hermetz, S. Wintle, R. Bhatia, P. de Vena Franks, B. Chieng, and W.-

L. Allen. The authors have not received any funding or grants in support of the presented research or for the preparation of this work and have no declarations of potential conflicts of interest. Jeremy Fabes was a recipient of a National Institute of Health Research Academic Clinical Fellowship for the duration of this study.

Corresponding author: Jeremy Fabes, University of Plymouth, Plymouth, UK

E-mail: jeremy.fabes@nhs.net

Experimental and Clinical Transplantation (2021)
Conclusions: Saphenous venous pressure, provided by peripheral cannulation, provided a reliable, less invasive, and safer alternative to femoral central line insertion for determination of the transcaval pressure gradient during the anhepatic phase of liver transplant.

Key words: Central venous pressure, Hemorrhage, Inferior vena cava

\section{Introduction}

During liver transplant, the inferior vena cava (IVC) is either fully cross-clamped or partially clamped, in the piggyback technique, to allow excision of the native liver and graft implantation. The placement of this clamp leads to an interruption to IVC blood flow, the extent of which depends on surgical technique and clamp positioning, ${ }^{1}$ and consequently an increase in IVC pressure. This venous pooling diminishes cardiac output, necessitating increased vasopressor support to maintain perfusion pressure and end-organ oxygen delivery. The extent of this obstruction to IVC flow can be derived by measurement of the pressure gradient between the IVC and central venous pressure (CVP), defined here as the transcaval pressure gradient (TCPG). ${ }^{2,3}$ During liver transplant, the TCPG provides useful real-time information on the venous pressure gradient and venous congestion caused by the caval clamp and also that caused by the caval anastomosis.,3 This information permits adjustment of the side-biting vascular clamp to decompress the IVC if a piggyback technique has been employed, helps guide fluid management, and also identifies flow obstruction at the IVC anastomoses, which may cause graft dysfunction. We have recently shown that the TCPG during the anhepatic phase of transplant is independently associated with risk of postoperative 
acute kidney injury, ${ }^{1}$ highlighting the clinical importance of this measurement and the value of IVC decompression. However, calculating the TCPG necessitates the insertion of a femoral central line for the purpose of IVC pressure assessment. The insertion of a second central venous catheter (CVC) increases patient risks, including inadvertent femoral artery cannulation or trauma, which can result in pseudoaneurysm formation, retroperitoneal hematoma, trauma to the femoral nerve, and risk of catheterassociated infection. Among liver transplant recipients, retroperitoneal hemorrhage carries significant consequences due to coagulopathy and may occur in up to $1.3 \%$ of cases. ${ }^{4-6}$

A safe and reliable alternative to femoral central line insertion for pressure measurement is desirable considering the risks of insertion. An alternative to the insertion of CVCs to measure great vessel pressure is the use of peripheral venous catheters as a proxy. With the assumption that there is uninterrupted fluid communication between a peripheral venous site and a central vein, the peripheral venous pressure and CVP should correlate. ${ }^{7,8}$ Evidence is already available on the ability of peripheral venous pressure monitoring to accurately reflect the CVP in the upper limb in hepatic surgery, ${ }^{9}$ hepatectomy, ${ }^{10}$ and liver transplant. ${ }^{11}$ However, a number of factors can impact the correlation between peripheral venous pressure and CVP, including the size and site of peripheral cannulation, ${ }^{12,13}$ core temperature, ${ }^{14}$ airway pressure, ${ }^{15}$ and whether the CVP is above tissue pressure, ${ }^{11,16-18}$ thereby preventing vascular collapse.

Peripheral saphenous venous pressure monitoring may provide an alternative and present an opportunity to avoid the need for femoral venous access, reducing patient risk and providing cost savings. Here, we have investigated the correlation between the peripheral saphenous venous pressure and femoral venous pressure and the ability of the saphenous pressure to reflect changes in femoral pressure during liver transplant.

\section{Materials and Methods}

\section{Ethics}

Ethical approval was obtained from the Cornwall and Plymouth Research Ethics Committee (reference number 17/SW / 0294), and the study was sponsored by the Royal Free NHS Foundation Trust.

\section{Conduct of anesthesia}

General anesthesia was induced and maintained as per clinician preference. In addition to routine anesthetic monitoring, invasive blood pressure, urine output, and temperature were measured. Temperature control was achieved with an under-patient warming mattress, forced air warmer, and warmed intravenous fluids. Central venous cannulation of the right internal jugular with a quad-lumen CVC (with 12- to 18-gauge channels) and an 18-gauge single-lumen CVC to either femoral vein was performed with the use of ultrasound guidance to confirm intravascular placement and to limit complications. Peripheral cannulation with an 18-gauge cannula was performed in the appropriate vein below the knee to match the side of insertion of the femoral CVC. Typically, this entailed cannulation of a large visible vein above or below the ankle. The saphenous cannula and femoral CVC were used only for pressure analyses. All 3 venous pressures were transduced continuously to ensure appropriate waveforms.

Thromboembolic deterrent stockings (TEDS) were applied as normally performed to both legs, with care to create the smallest disruption possible to the TEDS on the cannulated leg to remove external sources of pressure at the saphenous cannula insertion site. Sequential pneumatic compression devices were applied; venous pressure data were sampled at least 1 minute after the compression cycle was completed (on either leg). Saphenous vein cannulae were removed at the end of the procedure unless there was an ongoing need for additional venous access.

\section{Data collection}

During each phase of transplant (presurgical, dissection, anhepatic, reperfusion, closure), data were recorded at 2 timepoints at least 5 minutes apart. Mean venous pressures from the internal jugular, femoral, and saphenous cannulae were recorded. Additional data on core temperature, positive end-expiratory pressure, peak and mean airway pressure, mode of ventilation, and tidal volume were recorded.

\section{Study outcomes}

The primary outcome of this study was the correlation between saphenous and femoral pressure across all surgical phases. Secondary outcomes included the correlation between the saphenous 
and femoral pressure for the individual surgical phases. Further outcomes were the correlations between the venous pressure gradients between the saphenous and CVP (saphenous-to-CVP) and the femoral and CVP (femoral-to-CVP) for each surgical phase.

\section{Power calculation}

This is the first study of its kind to examine venous pressures in these 2 vascular territories. However, comparable work on comparisons on other vascular territories (ie, upper limb vs CVP) in similar circumstances have generated a range of correlation coefficients, with the largest study demonstrating a correlation coefficient of $0.89 .{ }^{12} \mathrm{~A}$ power analysis was performed to calculate the required sample size based on the 2-tailed Pearson product-moment coefficient. Because this is computationally identical to the Spearman rank correlation coefficient, this method was unaffected by the underling distribution. To generate a $90 \%$ power and $5 \%$ significance level with a presumed population correlation of 0.6 , a sample size of 20 was required.

\section{Statistical analyses}

The distribution of data was assessed using the Kolmogorov-Smirnov test of normality with Lilliefors significance correction. When data were combined throughout surgery, all variables, including the venous gradients, were non-Gaussian other than the peak airway pressure and tidal volume $(P=.200$ for both cases). Within each surgical phase, the distribution of venous pressures and gradients were normal other than end-of-case femoral pressure. This was expected as there is a shift in the venous pressures between each phase.

Associations between physiological and ventilator variables and venous pressures were determined using Spearman rank correlation coefficients for the pan-surgical data and Pearson correlation for within each surgical phase. To determine the independent effect of each variable on the saphenous venous pressure correlation, multivariable linear regression with backward elimination and main effects modeling were performed. Covariates included the femoral venous pressure, CVP, temperature, ventilator variables, and surgical technique. Model stringency used a probability of $F$ threshold of 0.1 for variable removal. All statistical analyses were performed using SPSS version 20 software.

\section{Inclusion and exclusion criteria}

Patients over the age of 18 years undergoing any form of primary and sole liver transplant at the Royal Free Hospital were eligible for this study.

Those patients in whom internal jugular or femoral CVC placement was not possible or who had significant complications from insertion were excluded. Additional exclusion criteria included the presence of concerns regarding vascular anatomy either preoperatively (ie, known significant varicose veins, previous deep venous thrombosis in any limb, skin infection, or prior radiotherapy of the cannula insertion site) or because of challenging cannulation with hematoma or multiple vessel perforation. Patients with known significant valvular disease or left ventricular failure (left ventricular ejection fraction $<40 \%$ ) were also excluded.

\section{Results}

\section{Study population}

Thirty patients were consented for this study over a period of 8 months; of these, 26 underwent liver transplant, and data collection was performed for 22 patients. No patients were excluded. All organ donations were from deceased donors.

\section{Primary outcome}

Combined analysis of data from all surgical phases demonstrated a reasonable correlation between the saphenous and femoral venous pressures $\left(\mathrm{r}^{2}=0.491\right.$, $P<.001$; Figure 1).

Figure 1. Saphenous Versus Femoral Venous Pressure Correlation for Al Surgical Timepoints

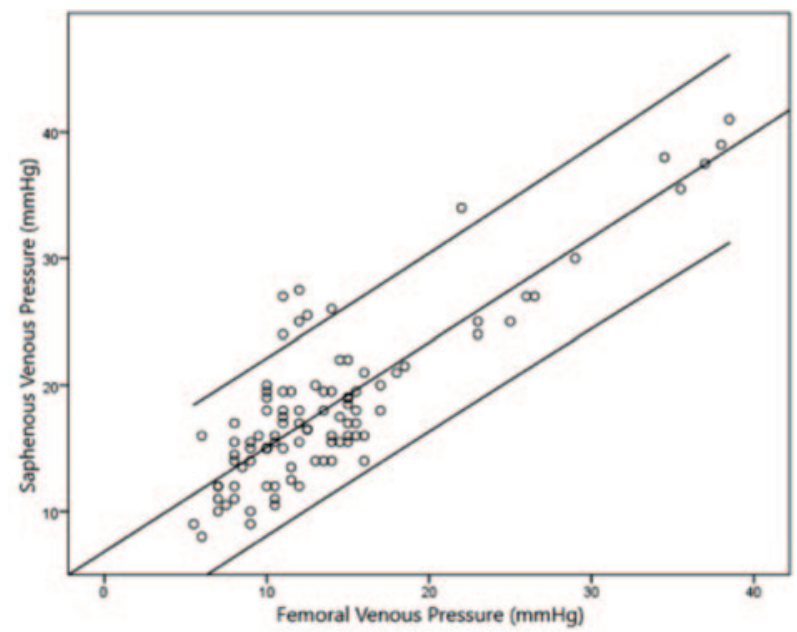

Figure shows linear line of best fit with individual 95\% confidence intervals 
The association between the saphenous and femoral pressures across all data points was determined from the following equation: saphenous venous pressure $=0.83 \times$ femoral venous pressure + $6.82 \mathrm{~mm} \mathrm{Hg}$. Table 1 shows that lesser degrees of correlation were seen between the peak airway pressure and the femoral and saphenous venous pressures. Mean airway pressure and CVP correlated with the femoral venous pressure only.

Multivariate analysis showed that the only independent association with saphenous venous pressure was femoral venous pressure (semi-partial correlation coefficient of $0.875, P<.001$ ), with all other physiological variables eliminated.

\begin{tabular}{|c|c|c|c|c|}
\hline & \multicolumn{2}{|c|}{$\begin{array}{c}\text { Saphenous } \\
\text { Venous Pressure }\end{array}$} & \multicolumn{2}{|c|}{$\begin{array}{c}\text { Femoral Venous } \\
\text { Pressure }\end{array}$} \\
\hline & $\begin{array}{l}\text { Correlation } \\
\text { Coefficient }^{\mathrm{a}}\end{array}$ & $P$ Value & $\begin{array}{l}\text { Correlation } \\
\text { Coefficient }^{\mathrm{a}}\end{array}$ & $P$ Value \\
\hline Femoral venous pressure & 0.701 & $<.001$ & & \\
\hline Temperature & 0.001 & .995 & 0.039 & .723 \\
\hline PEEP & -0.074 & .475 & 0.152 & .139 \\
\hline Peak airway pressure & 0.328 & .001 & 0.534 & $<.001$ \\
\hline Mean airway pressure & 0.173 & .092 & 0.282 & .005 \\
\hline Tidal volume & -0.013 & .902 & 0.123 & .233 \\
\hline CVP & 0.190 & .063 & 0.337 & .001 \\
\hline
\end{tabular}

Abbreviations: CVP, central venous pressure; PEEP, positive end-expiratory pressure

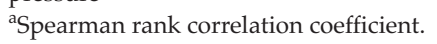

\section{Secondary outcomes}

The saphenous and femoral venous pressures during the anhepatic phase were highly correlated (Pearson $\left.\mathrm{r}^{2}=0.912 ; P<.001\right)$. Correlation was also good during all other surgical phases with the exception of the reperfusion phase (Table 2). A comparable analysis of the reperfusion phase using only cases that had cross-clamp or piggyback surgical approaches also did not demonstrate any correlation.

Table 3 shows the associations between the femoral-to-CVP and saphenous-to-CVP gradients throughout surgery. Correlation for the entire surgical episode was moderate (Spearman $\mathrm{r}^{2}=0.386$, $P<.001$; Figure 2). Correlations were excellent for the anhepatic, reperfusion, and closure phases but poor for the presurgical and dissection periods. The TCPG was significantly higher in the caval replacement surgical approach, compared with the piggyback approach, by $10 \mathrm{~mm} \mathrm{Hg}$ (femoral-to-CVP) and $7.4 \mathrm{~mm} \mathrm{Hg}$ (saphenous-to-CVP) in the anhepatic phase, as expected (Table 4).

Figure 3 presents the Bland-Altman plot for the difference between the saphenous and femoral readings across all surgical phases. A fairly tight distribution around the mean is shown with 5 readings (from 97 readings in total) outside the $95 \%$ confidence interval, which would be expected. The mean difference between the 2 readings was $4.4 \mathrm{~mm} \mathrm{Hg}$.

\begin{tabular}{|c|c|c|c|c|c|}
\hline \multirow[t]{2}{*}{ Surgical Phase } & \multicolumn{3}{|c|}{ Venous Pressure, $\mathrm{mm} \mathrm{Hg}$} & \multicolumn{2}{|c|}{$\begin{array}{l}\text { Femoral vs } \\
\text { Saphenous }\end{array}$} \\
\hline & CVP & Femoral & Saphenous & $\begin{array}{l}\text { Correlation } \\
\text { Coefficient }\end{array}$ & $P$ Value \\
\hline Whole case & 9.34 & 13.98 & 18.39 & $0.701^{b}$ & $<.001$ \\
\hline Presurgical & 8.89 & 10.32 & 16.89 & $0.834^{a}$ & $<.001$ \\
\hline Dissection & 8.55 & 10.52 & 14.86 & $0.522^{\mathrm{a}}$ & .015 \\
\hline Anhepatic & 7.75 & 20.85 & 24.07 & $0.955^{\mathrm{a}}$ & $<.001$ \\
\hline Reperfusion & 10.53 & 13.20 & 17.53 & $0.298^{a}$ & .202 \\
\hline Closure & 11.35 & 15.12 & 18.35 & $0.839^{a}$ & $<.001$ \\
\hline
\end{tabular}

Abbreviations: CVP, central venous pressure

apearson correlation coefficient. ${ }^{\mathrm{b}}$ Spearman rank correlation coefficient.

Table 3. Correlation Between Femoral-to Central Venous Pressure and Saphenous-to Central Venous Pressure Gradients

\begin{tabular}{|c|c|c|c|c|}
\hline \multirow[t]{2}{*}{ Surgical Phase } & \multicolumn{2}{|c|}{ Mean Gradient, mm Hg } & \multirow{2}{*}{$\begin{array}{c}\text { Correlation } \\
\text { Coefficient } \\
\text { (r) }\end{array}$} & \multirow[t]{2}{*}{ PValue $^{c}$} \\
\hline & $\begin{array}{c}\text { Femoral-to- } \\
\text { CVP }\end{array}$ & $\begin{array}{c}\text { Saphenous-to- } \\
\text { CVP }\end{array}$ & & \\
\hline Whole case & 4.64 & 9.05 & $0.621^{b}$ & $<.001$ \\
\hline Presurgical & 1.42 & 8.00 & $0.379^{a}$ & .110 \\
\hline Dissection & 1.98 & 6.26 & $0.708^{a}$ & .087 \\
\hline Anhepatic & 13.10 & 16.33 & $0.967^{a}$ & $<.001$ \\
\hline Reperfusion & 2.68 & 7.00 & $0.681^{a}$ & .001 \\
\hline Closure & 3.76 & 7.00 & $0.797^{a}$ & $<.001$ \\
\hline
\end{tabular}

Abbreviations: CVP, central venous pressure

${ }^{a}$ Pearson correlation coefficient. ${ }^{\mathrm{b}}$ Spearman correlation coefficient.

'Significance by analysis of variance, 2-tailed.

\begin{tabular}{|c|c|c|c|c|c|c|}
\hline & \multicolumn{3}{|c|}{ Femoral-to-CVP Gradient, $\mathrm{mm} \mathrm{Hg}$} & \multicolumn{3}{|c|}{ Saphenous-to -CVP Gradient, mm Hg } \\
\hline & $\begin{array}{c}\text { Caval } \\
\text { Replacement }\end{array}$ & Piggyback & Difference & $\begin{array}{c}\text { Caval } \\
\text { Replacement }\end{array}$ & Piggyback & Difference \\
\hline Presurgical & 1.17 & 1 & 0.17 & 7.5 & 7.83 & -0.33 \\
\hline Dissection & 2.36 & 1.58 & 0.78 & 5.64 & 7.12 & -1.47 \\
\hline Anhepatic & 19.5 & 9.54 & 9.96 & 21.08 & 13.69 & 7.39 \\
\hline Reperfusion & 3.42 & 1.85 & 1.57 & 7.33 & 6.46 & 0.87 \\
\hline End-of-case & 6 & 2.83 & 3.17 & 9 & 6.58 & 2.42 \\
\hline
\end{tabular}

Abbreviations: CVP, central venous pressure 
Figure 2. Saphenous to Central Venous Pressure and Femoral to Central Venous Pressure Gradient Correlation for All Surgical Timepoints

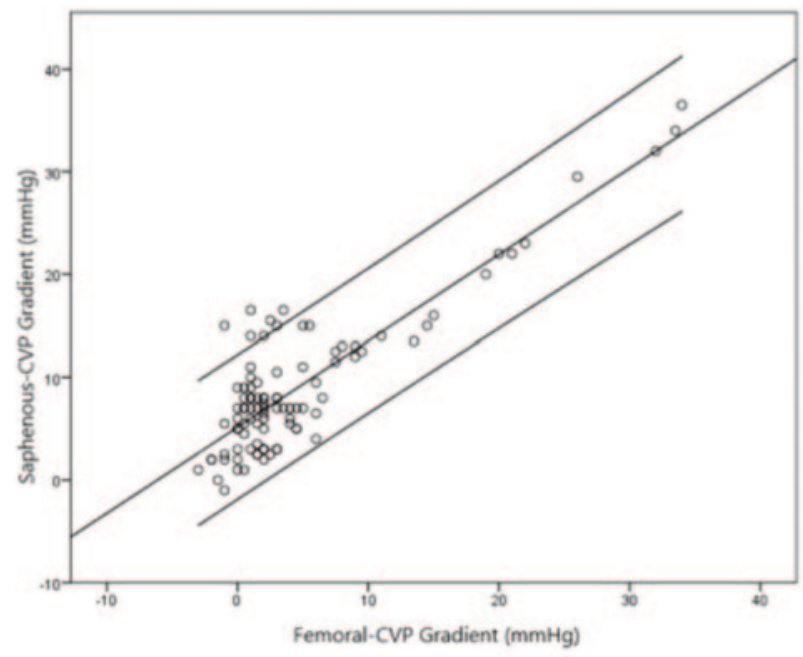

Abbreviations: $\mathrm{CVP}$, central venous pressure

Figure shows linear line of best fit with individual 95\% confidence intervals.

Figure 3. Bland-Altman Plot for Saphenous Versus Femoral Pressure Differences

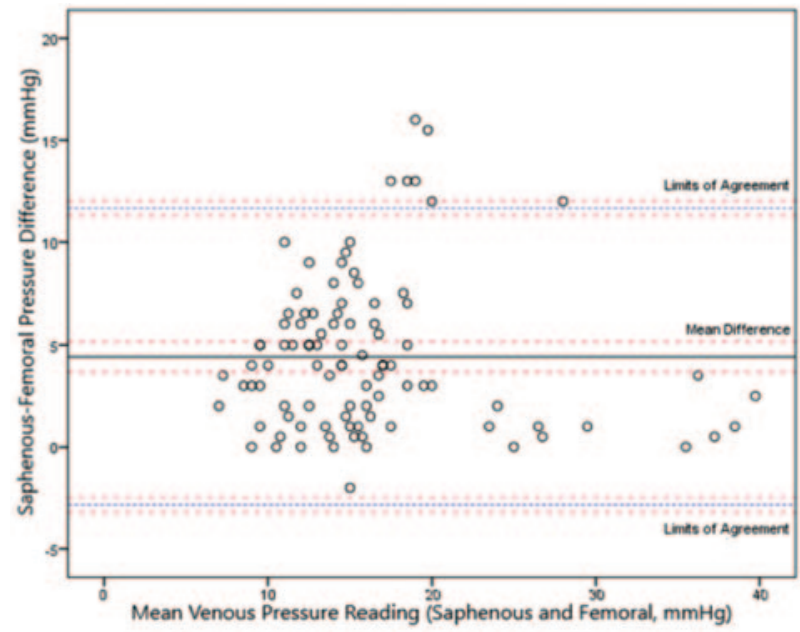

\section{Discussion}

\section{Main findings}

We demonstrated a tight correlation between the femoral and saphenous venous pressures during the anhepatic phase of liver transplant. This association was maintained within each surgical phase with the exception of the reperfusion phase. This association persisted when the CVP was taken into account, demonstrating that the saphenous-to-CVP gradient provides a reliable surrogate for the femoral-to-CVP gradient when assessing the TCPG. Furthermore, we demonstrated that the association between the saphenous and femoral venous pressures was maintained despite the presence of physiological and ventilatory variables.

The etiology of the divergence between femoral and saphenous pressures during the reperfusion phase (Table 2) is unclear. One explanation may include an alteration in peripheral venous tone as a consequence of hepatic ischemia-reperfusion injury. ${ }^{19-21}$ Venodilatation, with peripheral venous pooling, would drive a loss of correlation between CVP and peripheral venous pressure. Furthermore, the altered volume status during reperfusion may enhance the venous compression produced by the TEDS, resulting in venous compression, a loss of fluid column continuity, and a loss of correlation. Reperfusion is also the phase of surgery where the highest dose of vasopressors is required to maintain an acceptable blood pressure. The venoconstricting effect of noradrenaline and vasopressin may also cause a divergence in peripheral venous pressure and CVP. Despite the reduced correlation seen in the reperfusion phase, the value of a raised saphenous pressure would persist as a marker of a raised venous pressure gradient and the possibility of a surgical obstruction to venous return (ie, caval torsion or poor anastomotic flow).

We observed good femoral-to-CVP and saphenous-to-CVP correlations except for those shown in the presurgical and dissection phases $(P=.110$ and .087 , respectively). This may be as a result of variability in the extrinsic compression of the vena cava caused by the presence of ascites. The transplant recipients were a heterogenous group of patients with a variety of liver disease etiologies, many had significant portal hypertension and variable degrees of ascites. Ascites can cause extrinsic compression of the IVC at the start of surgery, prior to breaching of the peritoneum, which will affect the correlation. When ascites are drained during the dissection phase, this extrinsic compression, and hence variable obstruction to venous return, is removed.

\section{Limitations of this study}

The extrinsic compression derived from the TEDS and sequential pneumatic compression devices may have affected the saphenous pressures. It was noted that, when TEDS were particularly tight, the gradient from the saphenous to the femoral venous pressure was greater, although still consistent throughout the case. We did not collect data to reflect the intravascular 
status of the patients, which, as described above, may have contributed to the variability in venous pressures.

\section{Conclusions}

Operations involving manipulation or crossclamping of the IVC can result in significant hemodynamic instability; hence, knowledge of the degree of interruption to IVC flow is useful for patient management during these surgeries. For measurement of IVC pressure, a CVC inserted into the femoral vein is the gold standard; however, the practice of inserting femoral lines into coagulopathic patients involves risks that have significant implications should complications occur. Knowledge of the infrahepatic IVC pressure and hence the transcaval gradient, IVC to CVP, can assist in anesthetic management of liver transplant recipients. It can allow anesthetists insight into the cause of hypotension, giving the opportunity to differentiate between hypovolemia and surgical manipulation restricting venous return.

Knowledge of the transcaval gradient during the anhepatic phase can give anesthetists the opportunity to inform surgeons of the hemodynamic consequences of the IVC side-biting vascular clamp placement in the piggyback technique. This should result in the repositioning of the clamp, where it is surgically possible to do so, with potential hemodynamic benefits that are likely to impact on renal outcomes. ${ }^{1}$ There are some specific patient groups where maintenance of unobstructed venous return throughout the transplant is of critical importance. Patients with significant diastolic dysfunction (frequently seen in cirrhotic cardiomyopathy) or obstructive pathologies, like hypertrophic obstructive cardiomyopathy or inducible systolic anterior motion of the mitral valve, are very sensitive to alterations in preload. Knowledge of the IVC pressure in this cohort is useful so that care can be taken with both surgical technique and fluid management to optimize venous return.

We have demonstrated that transduction of the saphenous venous pressure from a peripheral cannula provided a reliable alternative to femoral central line insertion for determination of the transcaval venous gradient. This can allow clinicians to measure obstruction to venous return, which is especially relevant and modifiable in the anhepatic phase, in a less invasive and safer method than femoral venous cannulation.

\section{References}

1. Oliver CM, Fabes J, Ingram N, Rahman S, Krzanicki D, Spiro M; Royal Free Perioperative Research Group. Not all piggybacks are equal: a retrospective cohort analysis of variation in anhepatic transcaval pressure gradient and acute kidney injury during liver transplant. Exp Clin Transplant. 2021;19(6):539-544. doi:10.6002/ect.2021.0050

2. Parrilla P, Sanchez-Bueno F, Figueras J, et al. Analysis of the complications of the piggy-back technique in 1,112 liver transplants. Transplantation. 1999;67(9):1214-1217. doi:10.1097/00007890-199905150-00003

3. Navarro F, Le Moine MC, Fabre JM, et al. Specific vascular complications of orthotopic liver transplantation with preservation of the retrohepatic vena cava: review of 1361 cases. Transplantation. 1999:68(5):646-650. doi:10.1097/00007890199909150-00009

4. Kjellstrand CM, Merino GE, Mauer SM, Casali R, Buselmeier TJ. Complications of percutaneous femoral vein catheterizations for hemodialysis. Clin Nephrol. 1975;4(1):37-40.

5. Getzen LC, Pollak EW. Short-term femoral vein catheterization. A safe alternative venous access? Am J Surg. 1979;138(6):875-878 doi:10.1016/0002-9610(79)90314-3

6. Williams JF, Seneff MG, Friedman BC, et al. Use of femoral venous catheters in critically ill adults: prospective study. Crit Care Med. 1991;19(4):550-553. doi:10.1097/00003246-199104000-00016

7. Cox P, Johnson JO, Tobias JD. Measurement of central venous pressure from a peripheral intravenous catheter in the lower extremity. South Med J. 2005;98(7):698-702. doi:10.1097/01. SMJ.0000152543.10264.08

8. Tobias JD, Johnson JO. Measurement of central venous pressure from a peripheral vein in infants and children. Pediatr Emerg Care. 2003;19(6):428-430. doi:10.1097/01.pec.0000092586. 40174.2d

9. Stephan F, Rezaiguia-Delclaux S. Usefulness of a central venous catheter during hepatic surgery. Acta Anaesthesiol Scand. 2008:52(3):388-396. doi:10.1111/j.1399-6576.2007.01553.x

10. Choi SJ, Gwak MS, Ko JS, et al. Can peripheral venous pressure be an alternative to central venous pressure during right hepatectomy in living donors? Liver Transpl. 2007;13(10):14141421. doi:10.1002/It.21255

11. Hoftman N, Braunfeld M, Hoftman G, Mahajan A. Peripheral venous pressure as a predictor of central venous pressure during orthotopic liver transplantation. J Clin Anesth. 2006;18(4):251-255. doi:10.1016/j.jclinane.2005.09.031

12. Tugrul M, Camci E, Pembeci K, Al-Darsani A, Telci L. Relationship between peripheral and central venous pressures in different patient positions, catheter sizes, and insertion sites. J Cardiothorac Vasc Anesth. 2004;18(4):446-450. doi:10.1053/j.jvca.2004.05.022

13. Sherif L, Joshi VS, Ollapally A, Jain P, Shetty K, Ribeiro KS. Peripheral venous pressure as a reliable predictor for monitoring central venous pressure in patients with burns. Indian J Crit Care Med. 2015 Apr;19(4):199-202. doi:10.4103/0972-5229.154548

14. Sahin A, Salman MA, Salman AE, Aypar U. Effect of body temperature on peripheral venous pressure measurements and its agreement with central venous pressure in neurosurgical patients. J Neurosurg Anesthesiol. 2005;17(2):91-96. doi:10.1097/01.ana.0000158387.80678.bf

15. Kim SH, Park SY, Cui J, et al. Peripheral venous pressure as an alternative to central venous pressure in patients undergoing laparoscopic colorectal surgery. Br J Anaesth. 2011;106(3):305-311. doi:10.1093/bja/aeq399

16. Harvey MG, Cave G. Influence of tissue pressure on central venous pressure/peripheral venous pressure correlation: An experimental report. World J Emerg Med. 2011;2(2):93-98. doi:10.5847/wjem.j. 1920-8642.2011.02.003 
17. Cave G, Harvey M. The difference between peripheral venous pressure and central venous pressure (CVP) decreases with increasing CVP. Eur J Anaesthesiol. 2008;25(12):1037-1040. doi:10.1017/S0265021508004742

18. Kumar D, Ahmed SM, Ali S, Ray U, Varshney A, Doley K. Correlation between central venous pressure and peripheral venous pressure with passive leg raise in patients on mechanical ventilation. Indian J Crit Care Med. 2015;19(11):648-654. doi: 10.4103/09725229.169338
19. Seal JB, Gewertz BL. Vascular dysfunction in ischemia-reperfusion injury. Ann Vasc Surg. 2005;19(4):572-584. doi:10.1007/s10016-0054616-7

20. Kristiansen SB, Sheykhzade M, Edvinsson L, Haanes KA. Changes in vasodilation following myocardial ischemia/reperfusion in rats. Nitric Oxide. 2017:70:68-75. doi:10.1016/j.niox.2017.09.002

21. Jeong SM. Postreperfusion syndrome during liver transplantation. Korean J Anesthesiol. 2015;68(6):527-539. doi:10.4097/kjae.2015. 68.6.527 